\title{
OBSERVAÇõES SOBRE O ENCONTRO DE TRIPANOSSOMATÍDEOS DO GENERO BLASTOCRITHIDIA, INFETANDO NATURALMENTE TRIATOMÍNEOS EM INSETÁRIO E NO CAMPO
}

\author{
Eduardo Olavo da Rocha e Silva: \\ Dino Baptista Germano Pattoli** \\ Renato de Robert Corrêa *** \\ José Carlos Rehder de Andrade***
}

RSPU-B/341

Rocha E Silva, E. O. da et al. Obsrevaçōes sobre o encontro de tripanossomatideos do gênero Blastocrithidia, infetando naturalmente triatomineos $\mathrm{em}$ insetário e no campo. Rev. Saúde públ., S. Paulo, 11:87-96, 1977.

REsumo: Comprovada a presença de tripanossomatideos, não pertencentes ao gênero Trypanosoma, no tubo intestinal dos triatomíneos, o serviço especializado no combate desses insetos hematófagos vetores (SUCEN) da Secretaria da Saúde do Estado de São Paulo (Brasil), passou a adotar como rotina em seus laboratórios, a coloração do material fecal dos exemplares infetados por flagelados. Relata-se os resultados alcançados com a introdução dessa medida, destacando-se os primeiros achados em nosso meio, no campo, do P. megistus e T. sordida, encontrados infetados por flagelados do gênero Blastocrithidia.

UNITERMos: Tripanossomiase americana. Epidemiologia. Blastocrithidia. Triatomineos.

\section{I $N$ T R O D U C A O}

$O$ recente encontro em triatomíneos, de tripanossomatídeos não pertencentes ao gênero Trypanosoma Gruby, 1843 (Cerisola e cols. ${ }^{2}, 1971$ e Rocha e Silva e Ama$\mathrm{ral}^{6}, 1971$ ), abriu novas áreas de estudo e passou a exigir maior cuidado na identificação das formas flageladas, encontradas nos transmissores da doença de Chagas.

Em conseqüência, o órgão da Secretaria da Saúde do Estado de São Paulo en- carregado do combate aos vetores dessa endemia - Superintendência de Controle de Endemias (SUCEN), introduziu como rotina em seus laboratórios, a coloração das lâminas dos triatomíneos capturados no campo que. através do exame a fresco, apresentam formas flageladas no material fecal.

A presente comunicação tem por objeto chamar a atenção para os resultado: alcançados com a adoção desta norma.

* Da Superintendência de Controle de Endemias (SUCEN) da Secretaria da Saúde do Estado de São Paulo - Rua Tamandaré, 649 - São Paulo, SP - Brasil e do Departamento de Epidemiologia da Faculdade de Saúde Pública da USP - Av. Dr. Arnaldo, 715 - São Paulo, SP - Brasil.

* Do Departamento de Epidemiologia da Faculdade de Saúde Pública da USP.

** Da Superintendéncia de Controle de Endemias (SUCEN) da Secretaria da Saúde do Estado de São Paulo. 
ROCHA E SILVA, E. O. da et al. - Observações sobre o encontro de tripanossomatídeos do gênero Blastocrithidia, infetando naturalmente triatomíneos em insetário e no campo. Rev. saúde públ., S. Paulo, 11:87-96, 1977.

\section{MATERIAL E METODOS}

Nos laboratórios da SUCEN, o material intestinal dos triatomíneos, inicialmente, é examinado ao microscópio a fresco em gota de soro fisiológico, utilizando-se um aumento de 400 vezes. Notada a presença de formas flageladas, uma parcela desse material será misturada com gotas de soro humano inativo (fixador), posta a secar e corado pelo May-Grünwald-Giemsa. A seguir, as formas flageladas presentes nas lâminas serão estudadas com a objetiva de imersão $(1000 \times)$.

Foi assim que se obteve o material apresentado, cuja procedência se relata abaixo:

a) Exemplares do Triatoma infestans e Panstrongylus megistus, procedentes de colônias de insetário (Moji Guaçu, Estado de São Paulo), utilizados na realização de xenodiagnósticos em animais. Após o xenodiagnóstico, as caixas contendo os triatomíneos retornam ao insetário, onde os exemplares permanecem isolados, sem nova alimentação, aguardando a data dos exames $(30$ e 45 dias após o repasto-xeno).

b) exemplares capturados pelas equipes da SUCEN, no decorrer das atividades rotineiras de busca de focos de triatomíneos, nas casas e seus anexos.

\section{RESULTADOS}

No material examinado, cuja procedência foi esclarecida acima, observou-se em ocasióes diversas, o seguinte:

3.1 Flagelados (Figs. 1 a 4 ), identificados como sendo um tripanossomatideo monogenético, do gênero Blastocrithidia Laird, 1959 (Hoare ${ }^{4}, 1972$ ) que apresenta:

a) Formas epimastigotas $(=$ critidia $)$ com as extremidades geralmente afiladas; núcleo localizado no terço médio, lateralizado ou ocupando quase toda a largura do parasito. Cinetoplasto grande, arredondado ou alongado no sentido transverso do parasito, situado próximo ao núcleo. Flagelo livre, longo, relativamente grosso, apresentando ou não "strap-hangers" ou "banderillas" dispostas, no geral, em sentido único ao longo do flagelo.

Porém, o que mais chama atenção no flagelado são os vacúolos que se apresentam em tamanho e número variável, formando uma cadeia, em certos casos, ocupando quase todo o espaço situado entre o núcleo e a extremidade anterior do tripanossomatídeo. Na porção posterior ao núcleo, no entretanto, o citossomo apresenta-se homogêneo e no geral sem vacúolos.

b) Formas amastigotas ( $=$ leishmania) ovaladas ou arredondadas, outras vezes alongadas, com uma das extremidades mais afiladas que a outra. Essas formas são encontradas, encistadas ou não, livres ou então presas no flagelo (strap-hangers). Quando visível, o núcleo é relativamente grande e lateralizado. 0 cinetoplasto redondo, se situa mais ou menos afastado do núcleo.

c) Com muito menor freqüência, outras formas são observadas, como por exemplo, certas formas flageladas arredondadas (esferomastigostas?).

Varia muito o número de parasitas observados nos exemplares contaminados, desde o encontro de formas isoladas até as infecções maciças, nas quais, as formas epimastigotas se apresentam em grandes conglomerados ou florões. Quanto a hiometria da Blastocrithidia dos triatomíneos do insetário de Moji Guaçu, observe-se a Tabela 1 , onde se compara as medidas obtidas por Cerisola e cols. ${ }^{2}$ com as realizadas com o nosso material.

Através da microscopia ótica comum, essas formas muito se assemelham às da Blastocrithidia triatomae Cerisola e cols. ", 
ROCHA E SILVA, E. O. da et al. - Observações sobre o encontro de tripanossomatídeos do gênero Blastocrithidia, infetando naturalmente triatomíneos em insetário e no campo. Rev. Saude pübl., S. Paulo, 11:87-96, 1977.

1971. Quanto às medições realizadas, exceção do índice nuclear, elas se situam entre as máximas e mínimas da $B$. triatomae. Diferem no entanto da $B$. triatomae galvãoi, descrita por Carvalho ${ }^{1}$ (1973), pela presença dos vacúolos.

Albergando o flagelado em pauta, além do $T$. infestans já relatado por Rocha e
Silva e Amaral ${ }^{6}$ (1971), foram observados exemplares de outras espécies de triatomíneos, presentes em algumas colônias do insetário de Moji Guaçu, tais como: Triatoma sordida, Panstrongylus megistus e Panstrongylus lignarius.

A multiplicidade da contaminação, faz supor uma boa adaptação deste flagelado
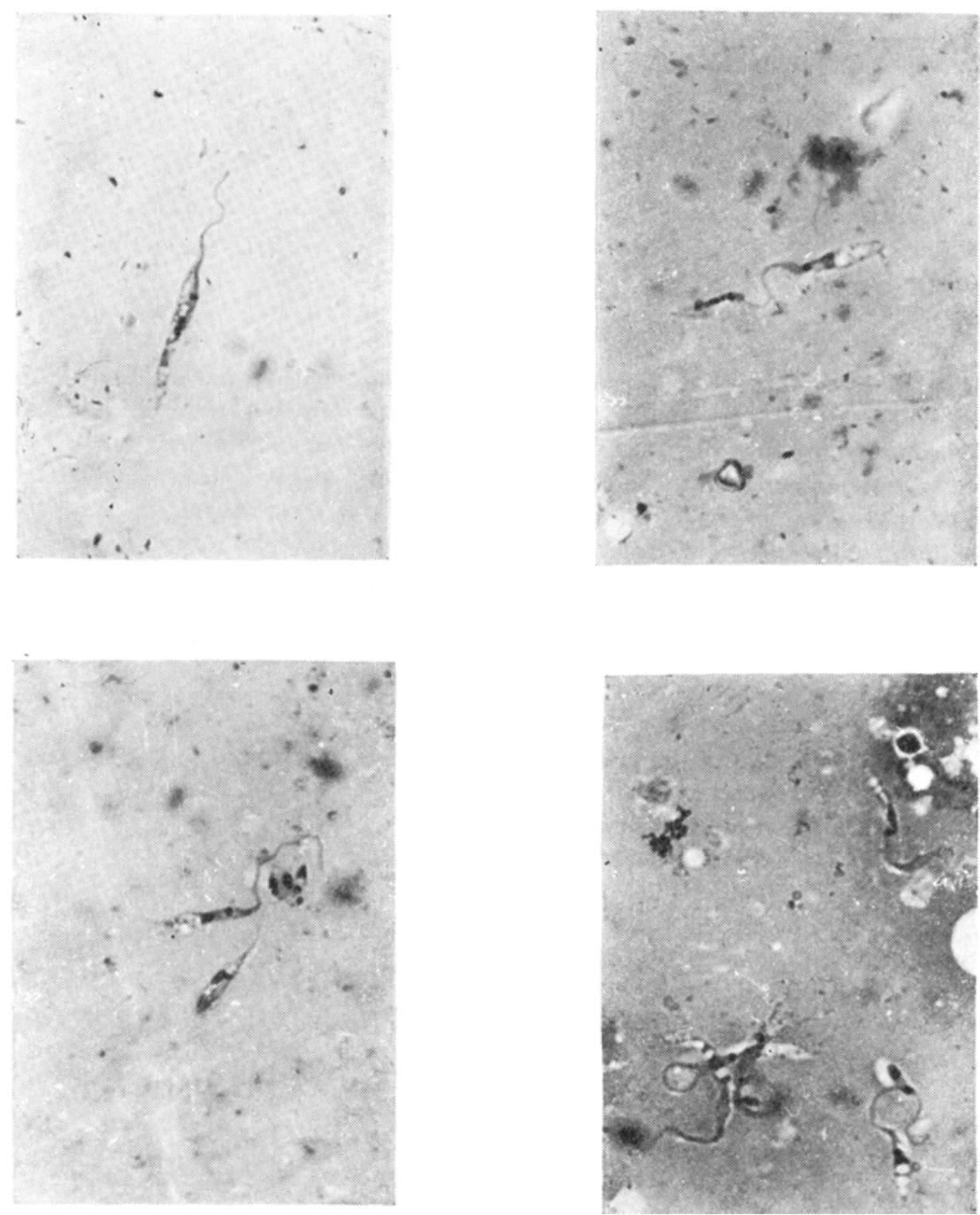

Figuras 1 a 4 - Flagelados encontrados em algumas colônias de triatomíneos — insetário de Moji Guaçu, SP (SUCEN) - semelhantes a Blastocrithidia triatomae. 

ROCHA E SILVA, E. O da et al. - Observacōes sobre o encontro de tripanossomatideos do gé. nero Blastocrithidia, infetando naturalmente tratomineos em insetário e no campo. Rev. saíle pribl, S. Paulo, 11:87-96, 1977.

\section{TABLA 1}

Dimensões da Blastochthdia triatomae e da Blastocrithuda dos triatomineos do insetário de Moji Guacu, Estado de São Paulo

\begin{tabular}{l|c|c}
\hline \multicolumn{1}{c}{ Nedidas (em microns) } & $\begin{array}{c}\text { Blastocrithidia } \\
\text { triatomae (*) }\end{array}$ & $\begin{array}{c}\text { Blastocrithidia de } \\
\text { Moji Guaçu (**) }\end{array}$ \\
\hline \hline Comprimento total (corpo + flagelo) & 53,5 & 51,8 \\
Corpo: & & 23,9 \\
$\quad$ Comprimento & 25,0 & 2,8 \\
largura (na altura do núcleo) & 2,6 & 23,3 \\
Elagelo livre & 22,5 & 11,7 \\
Núcleo à extremidade anterior \\
indice nuclear
\end{tabular}

\footnotetext{
(*) Cerisola, J.A. e cols : (1971)

(**) média
}

monogenético às condiçôes ambientais vigentés no referido insetário, principalmente. se considerarmos que ela rem se processando há aproximadamente cinco anos. Apresenta oscilações em torno dos $10 \%$ de exemplares infetados. em cada cristalizador contaminado. Este número parere variar em função de fatores vários. dos quais destacamos: época do ano e intervalo entre os repastos sanguíneos (aves) dos triatomíneos. Saliente-se, por outro lado. não ter sido notada, até então, variação sensível na mortalidade de exemplares das colonias contaminadas. se comparada com as não contaminadas.

3.2 Infecções mistas, ou seja, presença no material intestinal proveniente de un mesmo exemplar. de formas flageladas do gênero Blastocrithidia ao lado de fumas típicas do gênero Trypanosoma 1Figs. 5 e 61 . Constatou-se tal fato. entre alguns poucos exemplares do $T$. infestuns \& $P$. megistus. quando da realização de senodiagnósticos em animais. $\mathrm{Em}$ consequiência. a leitura dos xenos. em Moji Cuaçu. apresenta as seguintes alterniaijas de resultados:
- xeno negativo para tripanossomatídeos:

- xeno positivo para flagelados do gênero Blastocrithidia;

- xeno positivo para flagelados do gênero Trypanosoma;

- xeno positivo para Blastocrithidia e Trypanosoma.

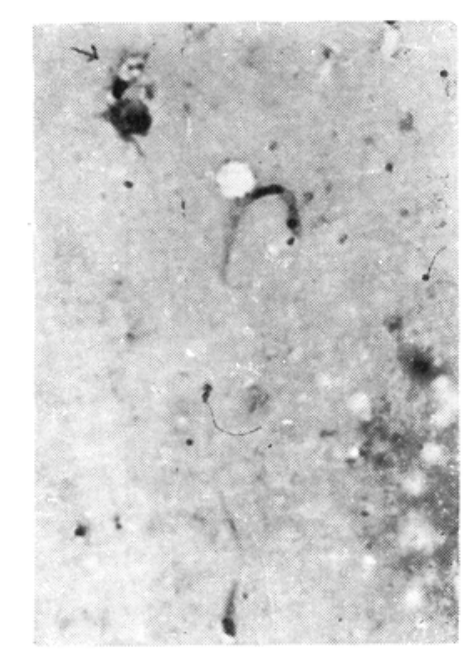

Fig. 5 - Fezes de triatomineo com forma tripomastigota do $T$. cru:l e forma epimast. gota da $B$. triatomae. 
ROCHA E SILVA, E. O. da et al. - Observaçōes sobre o encontro de tripanossomatídeos do gênero Blastocrithidia, infetando naturalmente triatomineos em insetário e no campo. Rev. Saúde públ., S. Paulo, 11:87-96, 1977.

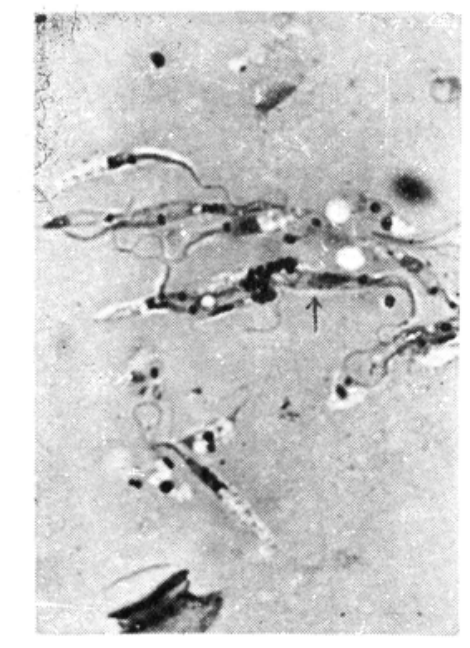

Fig 6 - Uma forma epimastigota do $T$. cruzi entre formas da $B$. triatomae.

Ressalta-se que nos raros casos de infecção dupla, observou-se predominância de formas da Blastocrithidia sobre Trypanosoma. Se isso resulta da presença de processo competitivo entre as espécies, é assunto em aberto e sem dúvida de interesse.

Por outro lado, se simplesmente nos encontramos diante de uma contaminação acidental das colônias pelas formas císticas primitivas do $T$. cruzi e formas delas derivadas, como suspeitou Silva ${ }^{\top}$ (1970) 71) ao se referir a igual achado de Cerisola e cols." é assunto a esclarecer.

A leitura desses xenos exige do examinador, não apenas bom conhecimento. mas também experiência e cuidadosa observação de múltiplos campos microscópicos. para a identificação correta das formas epimastigotas do Trypanosoma e das formas epimastigotas da Blastocrithidia.

3.30 encontro de tripanossomatideo monogenético do gênero Blastocrithidia. no tubo intestinal de $P$. megistus, capturado numa casa desabitada. do Bairro
Santo Antonio - município de São João da Boa Vista, reveste-se de significado. uma vez que demonstra a presença no campo, entre nós, de triatomíneos parasitados por tripanossomatídeos próprios de outros insetos que, iradvertidamente, poderão ser considerados como $T$. cruzi, se não forem tomadas as medidas aqui preconizadas ou equivalentes.

As Figuras 7 a 10 mostram o flagelado encontrado no $P$. megistus (ninfa do 5." estádio) que apresenta. supõe-se, aspectos morfológicos algo diferentes do tripanossomatídeo monogenético presente no insetário de Moji Guaçu.

As diferenças mais salientes, no caso. são: vacúolos menores. mais afastados do núcleo e em menor número. O citossomo é mais denso. As formas observadas apresentam maior comprimento e sua largura. na altura do núcleo, é também maior. O flagelo nas medidas realizadas é menor. Este tripanossomatideo, segundo dados comparativos contidos na Tabela 2, apresenta biometria algo diferente da Blastocrithidia triatomae, considerada como tal a presente no insetário de Moji Guaçu. Tendo em vista essas diferenças, aventouse a hipótese de se tratar de uma nova espécie.

No sentido de dar uma idéia quantitativa do significado desse achado, observe-se o total de exemplares do $P$. megistus, capturados no campo e examinados em Moji Guaçu, em 1972 (Tabela 3 ).

\section{DISC L S $\mathrm{SO}$}

A explanação feita. retrata nossa experiência com exemplares de triatomíneos infetados por tripanossomatídeos monogenéticos e permitem as seguintes considerações:

4. I No insetário de Moji Guaçu. foram encontrados exemplares do $T$. infestans. T. sordida. $P$. megistus e $P$. ligna- 
ROCHA E SILVA, E. O. da et al. - Observaçỏes sobre o encontro de tripanossomatídeos do gênero Blastocrithidia, infetando naturalmente triatomineos em insetário e no campo, Rev. Saúde públ., S. Paulo, 11:87-96, 1977.

rius, naturalmente infetados por um tripanossomatídeo monogenético semelhante a Blastocrithidia triatomae. Wallace" (1966) já chamara atenção para a pequena especificidade dos flagelados próprios dos insetos, sendo possivel a ocorrência de infecções cruzadas. até mesmo entre exemplares pertencentes a ordens zoológicas diversas.

Entre os reduviideos hematófagos, infecções desse tipo, no campo, certamente são raras. As possibilidades de contaminação aumentam, no entanto, quando certo número de triatomíneos são mantidos
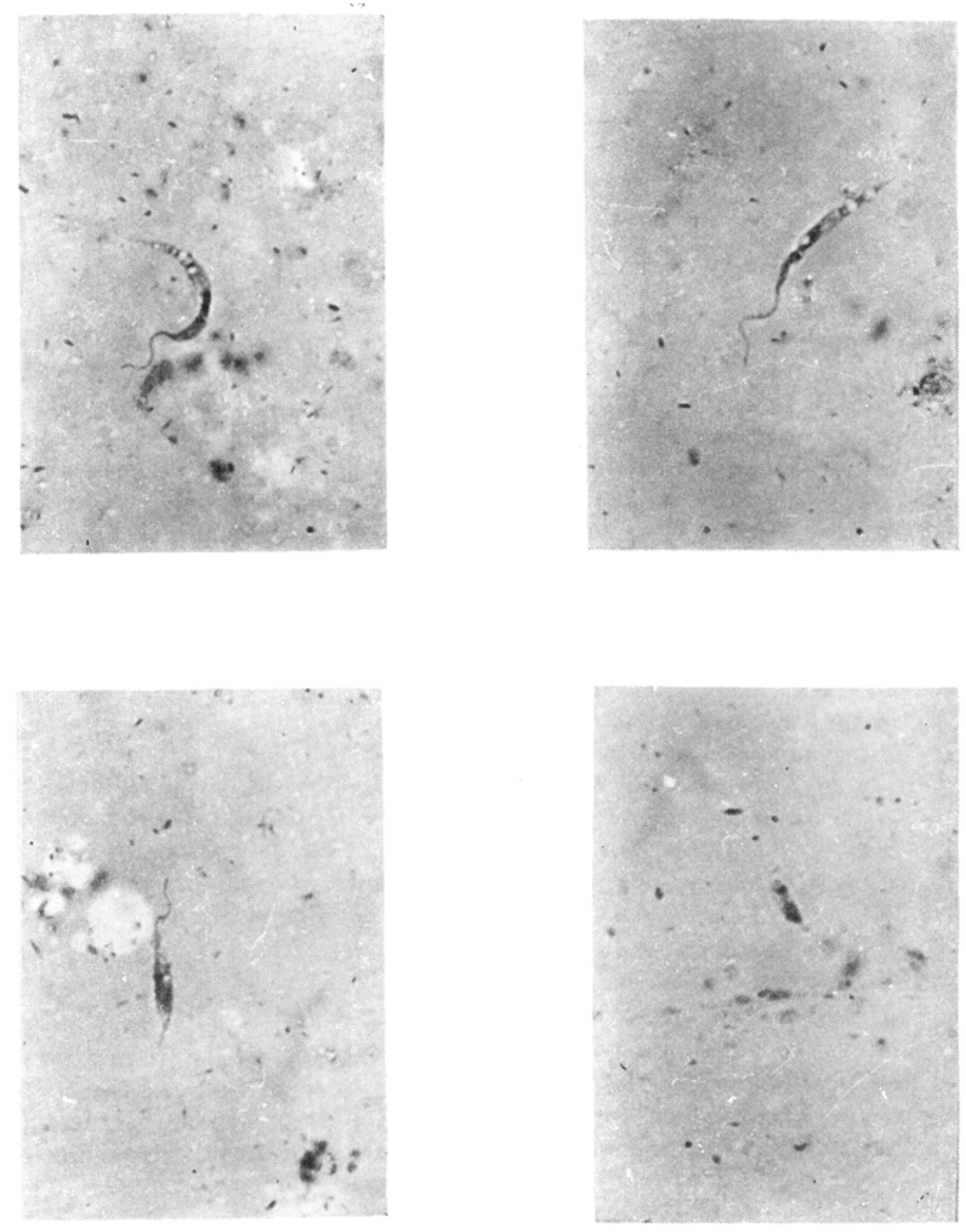

Figuras de 7 a 10 - Formas da Blastocrithidia sp. observadas no $P$. megistus, capturado numa casa desabitada. 
ROCHA E sILVA, E. O. da et al, - Observaçōes sobre o encontro de tripanossomatídeos do genero Blastocrithidia, infetando naturalmente triatomineos em insetario e no campo. Rev. Saúde públ., S. Paulo, 11:87-96, 1977.

TABEL 2

Dimensōes da Blastocrathidıa presente em triatomineos do insetárı de Mogı Guaşu. SP, e no Panstrongylus megistus do campo

\begin{tabular}{|c|c|c|}
\hline Medidas médias (em microns) & $\begin{array}{l}\text { Blastocrithidia } \\
\text { Moji Guaçu }\end{array}$ & $\begin{array}{l}\text { Blastocrithiadia } \\
\text { campo }\end{array}$ \\
\hline $\begin{array}{l}\text { Comprimento total (corpo }+ \text { flagelo) } \\
\text { Corpo: }\end{array}$ & 51.8 & 54,6 \\
\hline comprimento & 23,9 & 32,5 \\
\hline largura (na altura do núcleo) & 2,8 & 3,2 \\
\hline Flagelo livre & 23,3 & 18,6 \\
\hline Extremidade posterior ao núcleo & 11,7 & 13,1 \\
\hline Núcleo à extremidade anterior & 12,8 & 18,4 \\
\hline Indice nuclear & 0.91 & 0,71 \\
\hline
\end{tabular}

TABELA 3

Exames realizados nos exemplares do Panstrongylus megistus, capturados nas casas e anexos, situadas na área do Serviço Regional - 5. Campinas, Estado de S. Paulo, 1972

\begin{tabular}{|c|c|c|c|}
\hline \multirow{2}{*}{ P. megistus } & \multirow{2}{*}{$\begin{array}{l}\text { N. de exemplares negativos } \\
\text { para tripanossomatideos }\end{array}$} & \multicolumn{2}{|c|}{ N.o de exemplares positivos para } \\
\hline & & Trypanosoma & Blastocrithidia \\
\hline Ninfas & 3.214 & 283 & 1 \\
\hline Alados & 410 & 5 & - \\
\hline Total & 3.624 & 288 & 1 \\
\hline
\end{tabular}

Fonte: SUCEN (SR-5, Campinas)

em áreas restritas, junto a fontes de infecção, como pode ocorrer nos insetários e/ou quando os repastos sanguíneos são espaçados, como ocorre com exemplares usados nos xenodiagnósticos. Não sendo estrito o hematofagismo desses hemípteros. nas situações descritas e em outras equivalentes, os triatomíneos tendem a praticar a coprofagia e possivelmente o canibalismo. o que evidentemente facilita o processo de contaminação.

4.2 Entre triatomíneos capturados no campo foram encontrados exemplares na- turalmente infetados por tripanossomatídeos do gênero Blastocrithidia. Observamos o $P$. megistus e. posteriormente, em outros laboratórios da SUCEN foram encontrados exemplares do $T$. sordida, também infetados por flagelados do citado gênero (Tabela 4).

Embora tratando-se de achado ocasional como mostra a Tabela 5 é inegável que o mesmo apresenta implicações de importância prática. 
ROCHA E SILVA, E. O. da et al. - Observacões sobre o encontro de tripanossomatídeos do gênero Blastocrithidia, infetando naturalmente triatomineos em insetário e no campo. Rev. Saude pübl., S. Paulo, 11:87-96, 1977.

TA B E L A 4

Tripanossomatídeos do genero Blastocrithida encontrados no tubo intestinal de exemplares do T. sordıda, capturados no Estado de São Paulo, no periodo de 1972 a 1975

\begin{tabular}{|c|c|c|c|}
\hline Ano & Triatomineo & Estádio & $\begin{array}{l}\text { Local da captura: } \\
\text { Municipio }- \text { Localidade }\end{array}$ \\
\hline 1972 & T. sordida & 5.0 estádio & Santana da Ponte Pensa - Sítio (não esclar.) \\
\hline \multirow[t]{3}{*}{1973} & $T$. sordida & alado, fêmea & Ituverava - Faz. Mata Jacob \\
\hline & $T$. sordida & alado, fêmea & Mira Estrela - Faz. Tadeu Borba \\
\hline & $T$. sordida & $40^{\circ}$ estádio $(*)$ & Palmeira D'Oeste - Sitio São Martins \\
\hline \multirow[t]{2}{*}{1974} & $T$. sordida & alado, fêmea $(* *)$ & Rubinéia - B.o Uniāo \\
\hline & T. sordida & $4.0^{\circ}$ estádio & São João das Duas Pontes - Faz B. Vista \\
\hline 1975 & T. sordida & $2 .^{\circ}$ estádio & Monções - B.o Córrego do Cachorro \\
\hline
\end{tabular}

Fonte: SUCEN (Diretoria de Orlentação Técnica da D.C.V.)

(*) exemplar com infecção dupla (Trypanosoma + Blastocrithidia)

(**) dois exemplares

TABELA 5

Resultado dos exames realizados nos exemplares do Triatoma sordida, capturados nas casas e anexos, das regionais situadas no planalto paulista, no período de 1972.1975

\begin{tabular}{|c|c|c|c|c|}
\hline \multirow{2}{*}{ Ano } & \multirow{2}{*}{$\begin{array}{c}\text { N.o de exemplares } \\
\text { examinados }\end{array}$} & \multirow{2}{*}{$\begin{array}{c}\mathrm{N} \cdot{ }^{\circ} \text { de exemplares } \\
\text { negativos }\end{array}$} & \multicolumn{2}{|c|}{ Positivos para: } \\
\hline & & & Trypanosoma & Blastocrithidia \\
\hline 1972 & 34.023 & 33.520 & 502 & 1 \\
\hline 1973 & 26.935 & 26.713 & $220(*)$ & $3(*)$ \\
\hline 1974 & 26.406 & 26.216 & 187 & 3 \\
\hline 1975 & 26.745 & 26.561 & 183 & 1 \\
\hline
\end{tabular}

Fonte: SUCEN (Diretoria de Orientaçāo Técnica da D.C.V.)

(*) 1 exemplar com infecção dupla

4.3 O exame microscópico, a fresco, do conteúdo intestinal dos triatomíneos, é insuficiente para o correto reconhecimento dos flagelados por ventura presentes. Aconselha-se pois, a fixação do material para o adequado estudo e correta identificação das formas observadas.

Aliás, Pessoa ${ }^{5}$ (1974), tendo em vista possível confusão com o $T$, rangeli, cha- ma atenção sobre a necessidade de uma correta identificação do tripanossomatídeo observado.

4.4 A presença de um flagelado monogenético do gênero Blastocrithidia, em algumas colônias do insetário de Moji Guaçu, não impediu até o momento, a utilização desses exemplares na realização de xenodiagnósticos em animais. As formas 
ROCHA E SILVA, E. O. da et al. - Observaçóes sobre o encontro de tripanossomatídeos do gênero Blastocrithidia, infetando naturalmente triatomíneos em insetário e no campo. Rev. Saúde públ., S. Paulo, 11:87-96, 1977.

flageladas, detectadas nos exames, devem no entanto ser perfeitamente identificadas, o que se consegue através da coloração e estudo do material fecal, inclusive inoculação experimental.

4.5 A constatação de infecções mistas (Blastocrithidia e Trypanosoma) entre reduzido número de triatomíneos, com nítida predominância do flagelado monogenético sobre o digenético, pode decorrer, entre outros fatores da existência de possível processo de competição entre as espécies.

4.6 Vale ressaltar finalmente que Hanson e cols. ${ }^{3}$ (1968), já haviam chamado a atenção dos pesquisadores para o fato de que tripanossomatídeos, não pertencentes ao gênero Trypanosoma, poderiam vir a ser encontrados infetando triatomíneos, no campo, uma vez que essa possibilidade ficara demonstrada em condições de laboratório.
5. $\mathrm{CON}$ C L U S $\AA$

Entre exemplares de triatomíneos encontrados no campo, constatou-se a presença, no tubo intestinal, de formas flageladas pertencentes a tripanossomatídeos monogenéticos, identificadas como sendo do gênero Blastocrithidia.

Embora em se tratando de achado ocasional, sugere-se que medidas adequadas a melhor identificação dos flagelados sejam tomadas, rotineiramente, quando se tratar de exames realizados com exemplares procedentes do campo.

\section{A G R A D E I M E N TOS}

Aos senhores José de Campos Camargo, Antenor Nascimento Ferraz Filho e João Teixeira da Cunha, pela colaboração prestada.

RSPU-B/341

Rocha E Silva, E. O. da et al. [Observations on some tripanosomatides from genus Blastocrithidia, naturally infecting triatomines in the laboratory and the field.] Rev. Saúde públ., S. Paulo, 11:87-96, 1977.

ABSTRACTS: In confirming the presence of tripanosomatides not belonging to the Trypanosoma genus in the triatomes' intestine induced SUCEN (Secretaria da Saude do Estado de São Paulo) to the routine staining of slides of the collected material in the intestinal tube of bugs for the identification of the flagellated shapes. The results obtained with the introduction of this technique and the first discovery of Panstrongy megistus and Triatoma sordida, in São Paulo, with natural infection, in the field, by trypanosomatides of genus Blastocrithidia are reported.

UNITERMS: American trypanosomiasis. Epidemiology. Blastocrithidia. Triatomids.

\section{REFERENCIAS BIBLIOGRAFICAS}

1. CARVAlHO, A.L. de M. - Estudos sobre a posição sistemática, a biologia e a transmissão de tripanossomatídeos encontrados em Zelus leucogrammus. Belo Horizonte, 1973. Tese de mestrado. Univ. Fed. Minas Gerais, 1973.
2. CERISOLA, J.A. et al. - Blastocrithidia triatomae $\mathrm{n.sp}$. found in Triatoma infestans from Argentina. J. Protozool., 18:503-6, 1971.

3. HANSON, L.W. et al. - Experimental infection of Triatoma infestans and 
ROCHA E SILVA, E. O. da et al. - Observaçóes sobre o encontro de tripanossomatideos do gênero Blastocrithidia, infetando naturalmente triatomineos em insetário e no campo. Rev. Saúde públ., S. Paulo, 11:87-96, 1977.

Rhodnius prolixus with trypanosomatidae of the genera Crithidia and Blastocrithidea. J. Protozool., 15:31449.1968.

4. HOARE, C.A. - Classification in The Trypanosomes of Mammals. Oxford, Blackwell Scientific Publ., 1972.

5. PESSOA, S.B. \& MARTINS, A.V. - Trypanosomidae - Gênero - Trypanosoma. Tryparosoma (Schiszotripa. num) cruzl e Moléstia de Chagas. In: PESSOA, S.B. Parasitologia médica. 9.8 ed. Rio de Janeiro, Guanabara Koogan, 1974. p. 141-84.

6. ROCHA E SILVA, E.O. da \& AMARAL, A.D.F. do - Sobre o encontro de um parasita do genero Blastocrithi- dia em exemplares de $T$. infestans criados em colonias de laboratório: Nota prévia. Rev. paul. Med., S. Paulo, 78:92, 1971.

7. SILVA, I.I. - Como las formas primitivas del Trypanosoma (Schizotrypa. num) cruzi, fueron consideradas erróneamente una nueva especie. Rev. Fac. Mea. Tucumán, 10:219.37, 1970/ 71 .

8. WALlAGE, F.G. - The Trypanosomatid parasites of insects and arachnids. Exp. Parasitol., 18:124-93, 1966.

Recebido para publicação em 08/09/1976 Aprovado para publicaça em 10/09/1976 